\title{
Simulation Model, Warm-up Period, and Simulation Length of Cellular Systems
}

\author{
Samad Salehi Kolahi \\ School of Computing and Information Technology \\ Unitec New Zealand \\ Auckland \\ NEW ZEALAND
}

\begin{abstract}
In this paper, discrete event simulation by batch-means of a $\mathrm{M} / \mathrm{M} / \infty$ queuing system is utilised to simulate a cellular CDMA system. The details of the simulation model, warm-up period, and simulation run time are discussed. The warm-up period is studied because it affects the accuracy of the results in simulation of communication systems. During the warm-up period-when the simulation system has not reached the steady-state situation-, the system results (eg blocking probability) vary very rapidly from zero to 0.037 for the parameters used. In the batch-means method with 10,000 calls per batch and for 50 batches $(500,000$ calls), the CDMA blocking probability is 0.0192 with $99 \%$ confidence interval.
\end{abstract}

Key word: Simulation Model, warm-up period, stochastic simulation, CDMA, simulation run-time, blocking probability

\section{INTRODUCTION}

In stochastic simulation the input data (for example arrival and service times) are random variables, the output results might vary depending on the simulation length. Statistical behaviour and analysis of the random variables is therefore very important in determining the accuracy of the simulation results [1]. In addition, the simulation model is an abstract model and the results are not as deterministic as the hardware model's results.

During the last forty years, a variety of methods have been suggested for data collection and analysis of the non-stationary queuing processes. One of the pioneering papers that initiated much discussion was 'Some tactical problems in digital simulation' by Conway published in 1963 [2]. Conway highlighted that initially the queuing system is empty and it takes some time for the system to reach the steady-state situation. The initial stage is called the transient or warm-up period. Collecting data during this period could affect the accuracy of the results. Since Conway's paper, a variety of methods have been suggested to minimise the effect of the warm-up period and provide results that could be used with a certain confidence. In [24], analysis of results in a cellular environment was discussed. In this paper, the method of the development of a cellular simulation model is further discussed. The simulation warm-up period and run time, are further analysed in the batchmeans simulation method used. The organization of this paper is as follows. In next section, the simulation by batch means method is discussed. Section 3 covers system model. The simulation analysis results are then presented in Section 4. The last section presents conclusions.

\section{SIMULATION METHOD}

Several methods (regenerative simulations, replications method, and batch means method) have been suggested for data collection and analysis [3]. In regenerative simulation there is a state called the regenerative state that the system returns to, over and over again. The process starts anew probabilistically each time this state is visited. The main advantage of the regenerative simulation is that one does not need a warm-up period. In the method of replications, the simulation is repeated a number of times, each time with a different series of random numbers. Average values of the results are collected during each replication. In replication methods, each replication has its own warm-up period and this would reduce the accuracy of the results [4]. If a single long simulation run is used (batch-means method), the warm-up period needs to be determined and removed only once.

The batch-means method is used in this study to simulate the CDMA system. In this method, the results are obtained from a single simulation run time but the simulation observations are divided into batches [5]. The simulation is run for a number of batches and continued until the required precision is reached between the results of various simulation batches [3].

The observations from $K$ batches with $n$ observation per batch are

$$
\left(X_{11}, X_{12}, X_{13} \ldots \ldots \ldots \ldots . X_{1 n}\right),\left(X_{21}, X_{22}, X_{23} \ldots \ldots \ldots \ldots X_{2 n}\right), \ldots \ldots \ldots,\left(X_{K 1}, X_{K 2}, X_{K 3} \ldots \ldots \ldots \ldots X_{K n}\right)
$$

As the first batch has a warm-up period, and assuming $w$ observations belong to initial warm-up period, the mean value of results for the first batch $\left(\mu_{1}\right)$ is [3]

$$
\mu_{1}=\frac{1}{n-w} \sum_{j=1}^{n} X_{1 j}
$$

The mean value of results per batch, $\left(\mu_{2}\right), \ldots,\left(\mu_{n}\right)$ can be calculated as

$\mu_{i}=\frac{1}{n} \sum_{j=2}^{n} X_{i j}$

and the sample mean of the various iterations is

$\bar{\mu}=\frac{1}{K} \sum_{i=1}^{K} \mu_{i}$

so that $\bar{\mu}$ is the average of the averages. The sample variance for the iterations is thus 


$$
\sigma^{2}(K)=\frac{1}{K-1} \sum_{i=1}^{K}\left[\mu_{i}-\bar{\mu}\right]^{2 \cdots}
$$

Applying the Central Limit Theorem[6-8], for a very large number of call arrivals, it is fair to assume a normal distribution for $\mu_{i}$ with an estimated mean $\bar{\mu}$ and standard deviation $\sigma(\mathrm{k}) / \sqrt{\mathrm{K}}$. For a normally distributed variable, the $95 \%$ confidence interval is \pm 1.96 times the standard deviation of the mean and the $99 \%$ confidence interval is \pm 2.58 times the standard deviation of the mean [7]. If $\overline{\bar{\mu}}$ is the true mean, then the $99 \%$ confidence interval is

$$
P\left(-2.58 \frac{\sigma}{\sqrt{K}}<\overline{\bar{\mu}}-\bar{\mu}<+2.58 \frac{\sigma}{\sqrt{K}}\right)=0.99
$$

Hence the true mean $\overline{\bar{\mu}}$ is $\bar{\mu} \pm 2.58 \sigma(K) / \sqrt{K}$ with 99\% confidence and $\bar{\mu} \pm 1.96 \sigma(K) / \sqrt{K}$ with $95 \%$ confidence [9]. The difference $\overline{\bar{\mu}}-\bar{\mu}$ is the error of the estimate, the difference between the true mean and the estimated mean. If the results within a $1 \%$ margin are accepted, the following inequity should hold to terminate the simulation

$$
\frac{2.58 \sigma(K) / \sqrt{K}}{\bar{\mu}}<0.01
$$

Note that as $K$ (number of batches) increases, the LHS of the above inequality decreases. Essentially (7) is a test, the batches are continued until it is satisfied. In the CDMA simulation model, and for each batch, the mean value of the average blocking probability is obtained for 10000 call arrivals. When the number of call arrivals exceeds 10000, the second batch begins. The batches are continued until the required $99 \%$ confidence in the results is reached.

\section{SYSTEM MODEL}

A cellular CDMA system (a home cell and three tiers of neighbouring cells as discussed in [10]) is considered with a base station located at the centre of each cell. The signal strengths of three neighbouring cells are compared to find the home cell as found in [11]. Calls are considered to suffer log-normal shadowing (but not Rayleigh fading [12]). All cells are assumed to be homogeneous in every respect and users are assumed to be uniformly distributed over the cell area. The reverse link (mobile to base station) is investigated as it is the limiting link due to its inferior performance compared to the forward link [13].

The CDMA system is modelled as a $\mathrm{M} / \mathrm{M} / \infty$ system $[12,14]$. Calls are assumed to arrive in the system with Poisson distributed probability with mean arrival rate $\lambda$ calls/second. Calls remain in the system with a negative exponentially distributed call holding time with mean $1 / \mu$ seconds/call. When a call is accepted it remains in the system during its call holding time. There is no limit on the number of calls accepted to the system (soft capacity) as long as they meet the required call quality of $7 \mathrm{~dB}$. Any calls not meeting this required quality are blocked from entering the system.

Call quality in terms of the energy per bit over total interference spectral density $\mathrm{E}_{\mathrm{b}} / \mathrm{N}_{\mathrm{o}}$ may be calculated as [15]:

$$
\frac{E_{b}}{N_{0}}=\frac{S / R}{I / W}=\frac{S / R}{\left((N-1) S+I_{o}+\eta\right) / W}=\frac{W / R}{(N-1)+(I / S)_{o}+\eta / S}
$$

Where $(\mathrm{I} / \mathrm{S})_{0}$ is the ratio of other-cells interference to the received signal strength $(\mathrm{S})$ at the home base station, $\eta$ is background noise, $W / R$ is the Processing Gain, $\mathrm{W}$ is available spread bandwidth, $\mathrm{R}$ is data rate, and $N$ is the number of active users. Taking voice activity $(\alpha)$ into consideration,

$\frac{E_{b}}{N_{0}}=\frac{W / R}{\alpha(N-1)+\alpha(I / S)_{o}+\eta / S}$

The total other-cell interference $I_{o}$ is the interference produced by all users who are power controlled by other base stations. Assuming a CDMA system with $M$ outer cells and $N$ users per cell, then the total other user interferences-to-signal ratio $(I / S)_{o}$ is [12]:

$$
(I / S)_{o}=\sum_{i=1}^{M} \sum_{j=1}^{N} I_{i j} / S
$$

The system model is further explained in $[11,16]$

\section{A. Progressive Development and Results Verification of the Simulation Model}

The model was built (creating a $\mathrm{C}$ simulation software of the CDMA system) and tested progressively from a simple queuing model to a complex $M / M / \infty$ CDMA model to ensure its accuracy. The first model developed was a $\mathrm{M} / \mathrm{M} / 1$ queue with Poisson arrivals with exponential call holding times, single queue, single server, and no limit on the size of the queue. The average number of uses in the system is given by the formula $[17,18]$

$\bar{N}=\frac{\lambda}{\mu-\lambda}$

Using both the analytical and simulation method, for arrival rate $(\lambda)$ of 0.8 and call holding time $(1 / \mu)$ of 1 , the average number of calls in the system was found to be four. As the system has infinite size, there will be no call losses and probability of 
blockage is always zero. Next, the model was modified to a $M / M / 1 / K$ queuing model and the results were compared with the analytical results. This queue is similar to the previous $\mathrm{M} / \mathrm{M} / 1$ queue but the system size is limited to $k$ (including the call in service). The probability of a call arriving and finding the system queue full, and hence, the call "lost", is given by [18]

$$
P_{k}=\frac{1-\lambda / \mu}{1-(\lambda / \mu)^{k+1}}\left(\frac{\lambda}{\mu}\right)^{k}
$$

where $k$ is the system capacity, the total number of calls in the queuing system [9, 18]. The blockage probability can also be calculated using the $M / M / 1 / K$ simulation model where the system was put under different traffic loads, and the blockage probability was found by dividing the total number of blocked calls over the total number of call arrivals. For a given traffic load of $0.1, \lambda / \mu$, and the system size of $k=2$, the simulation blocking probability was found to be approximately 0.01 matching the result from the above formula. In the next step of the model development strategy, a more complex $M / M / K / K$ queuing model was built. Any calls that arrive and find all the servers busy are not queued and are lost (blocked-calls cleared). The blocking probability for a given traffic load, $\lambda / \mu$, can then be obtained by calculating the ratio of the total number of blockages over the total number of arrivals. For the traffic load of 10 Erlang $(\lambda=20, \mu=2)$ and for 12 circuits $(K=12)$, the simulation results provided a blocking probability of $P_{k}=0.12$. The blocking probability for a $M / M / K / K$ system can also be determined by Erlang-B formula $[19,20]$.

$$
P_{\text {blocking }}=\frac{(\lambda / \mu)^{K} / K !}{\sum_{k=0}^{K}(\lambda / \mu)^{k} / k !}
$$

where $\lambda / \mu$ is Erlang traffic (mean traffic capacity), and $K$ is the number of required circuits. For the traffic load of 10 Erlang, and $K=12$, the Erlang-B formula provides blocking probability of $\mathrm{Pk}=0.12$. Alternatively, the fixed telephone network traffic tables can be used to find the blocking probability. The analytical formula results of $\mathrm{P}_{\mathrm{k}}=0.12$ matched perfectly with the simulation results. Next, the simulation model was modified for a CDMA system. CDMA is a $M / M / \infty$ system where there is no limit on the number of servers (channels), calls will not get blocked because of the non-availability of a circuit as it was for a $M / M / K / K$ system. The call admission policy in the simulation model was changed in order to accept the calls if they meet the required quality of $7 \mathrm{~dB}$. In addition, the $M / M / K / K$ model was modified to allocate a mobile location and propagation parameters, choose a cell site, and gather the relevant call statistics described earlier. The simulation was then performed for a set of parameters. The CDMA simulation model results was verified in $[21,22]$ with analytical results obtained in [14].

In the simulation model, a circular queue (figures 1) was used to hold the cellular call arrivals. The size of the circular queue must be bigger than the total number of calls allowed in the system and this can be estimated through some initial simulation test runs. On each arrival the rear of circular queue is added by one and on every departure the front of the circular queue is added by one.

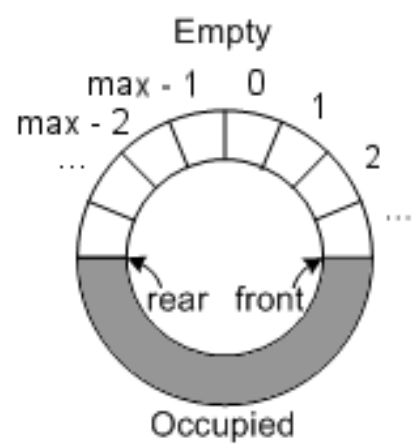

Figure 1: A circular queue

Figure 2 is the call arrival and departure to the queuing system. It is further explained in figure 3 flow chart. Each server is the processing of the call in cellular system.

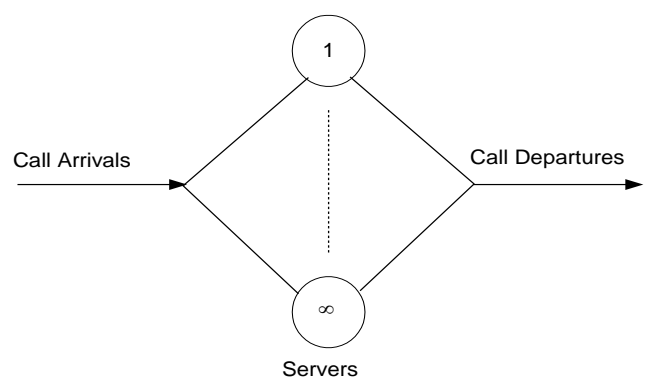

Figure 2: Call arrivals, service, and departures for $M / M / \infty$ system.

The step-by-step method used to build a complete cellular simulation model is fully explained in detail in [23].

\section{ANALYSIS OF SIMULATION WARM UP PERIOD AND SIMULATION LENGTH}

To study the effect of the initial warm-up period on the accuracy of the results, the simulation was performed under various scenarios for the CDMA parameters of $\mathrm{W} / \mathrm{R}=125$ (processing gain), $\alpha=0.4$ (voice activity), $\mathrm{Eb} / \mathrm{No}=7 \mathrm{~dB}$ (call quality), $\mathrm{S} / \eta=-1 \mathrm{~dB}$ (signal to background noise), $\sigma \xi=8 \mathrm{~dB}$ (shadowing parameter), and $m=4$ (distance exponent) and traffic load of 27 Erlangs. 
Initially, the simulation was performed with three different sets of random numbers for the first 700 call arrivals. The first few call losses (blocked calls) for the three simulations were call numbers $(62,98,106$, $113, \ldots)$ and $(79,88,98,202, \ldots)$ and $(124,318,327$, $379, \ldots)$. Due to randomness of the CDMA parameters, one cannot estimate which calls will be lost. In the three simulations, there were no call losses for the first 61,78 , and 123 calls respectively. Initially the system is empty and there is a low probability of call losses. As the system has not reached the steady state, there is a large variation in blocking probability and there is not much similarity between the three simulation results. The blocking probability varies between zero and 0.037 .

The simulation was also performed for two simulation runs with different random numbers, and for the first 10,000 call arrivals, and the blocking probability graph is shown in Figure 4. The warm-up period can be clearly seen in the graph where initially there is a low probability of blockage and data collected during this period should be ignored. As the number of calls increase, the randomness in the results decreases and the results of the two simulation runs approach the blocking probability of $0.018-0.020$.
In the batch means method used, 50 batches used with 10000 calls per batch $(500,000$ call arrivals) to obtain blocking probability of 0.0192 with $99 \%$ confidence interval.

\section{CONCLUSION}

Direct stochastic simulation by batch-means was used to simulate a CDMA system with there tiers of neighbouring cells. $\mathrm{M} / \mathrm{M} / \infty$ queuing system was used to simulate the CDMA system. Calls were accepted the system if they met the call quality of $7 \mathrm{~dB}$. The simulation results indicated that initially the system blocking probability varied rapidly from zero to 0.037 for the parameters used. Simulation warm-up period can affect the accuracy of the results and therefore it is important to accuracy determine the warm-up period to obtain the correct results. The simulation results and warm-up period was determined for simulation with 10,000 calls. In a batch-means method with 10,000 calls per batch and 50 batches $(500,000$ call arrivals), the blocking probability was 0.0192 with $99 \%$ confidence interval.

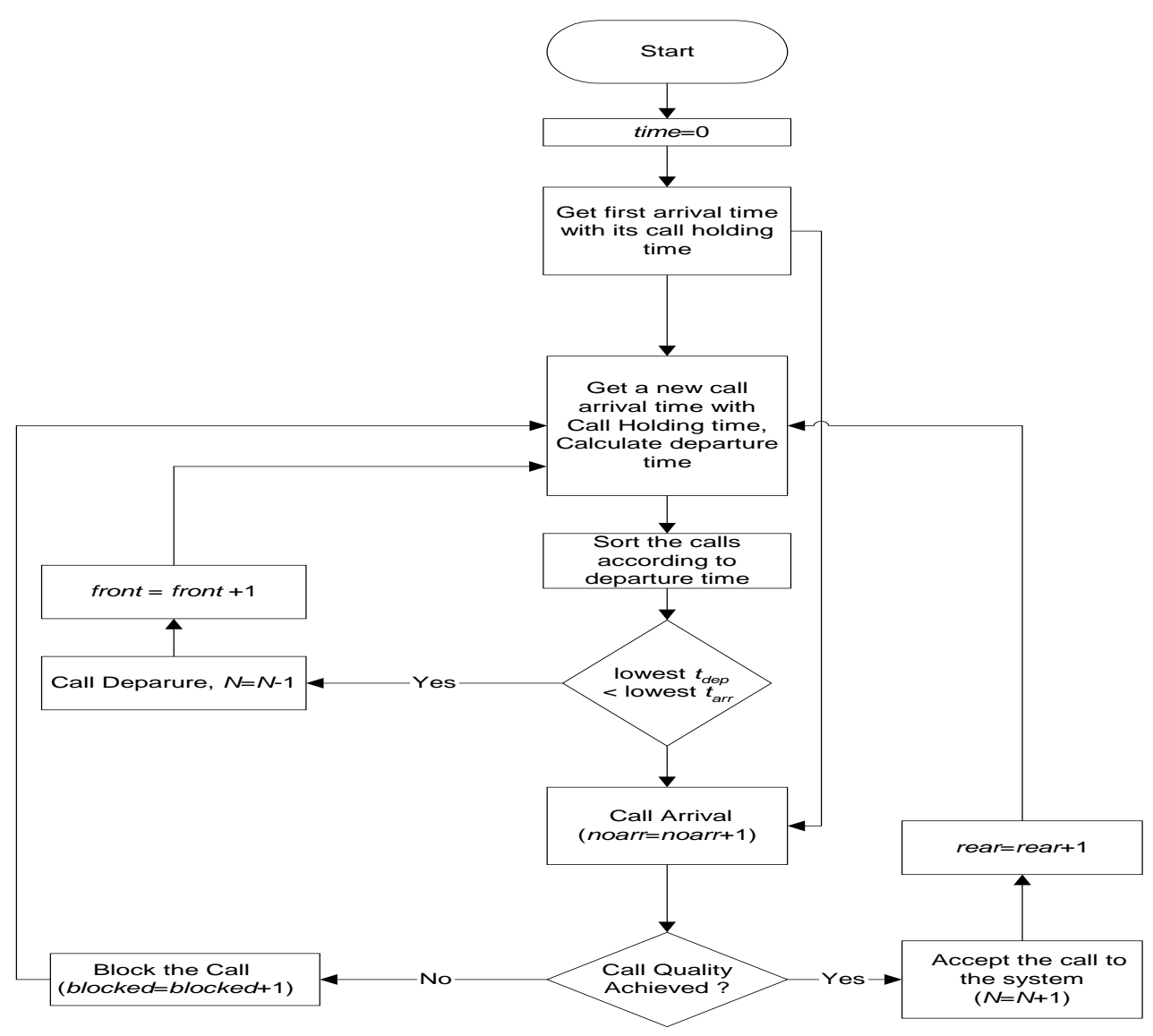

Figure 3: Call arrivals and departures flowchart for the cellular system. 


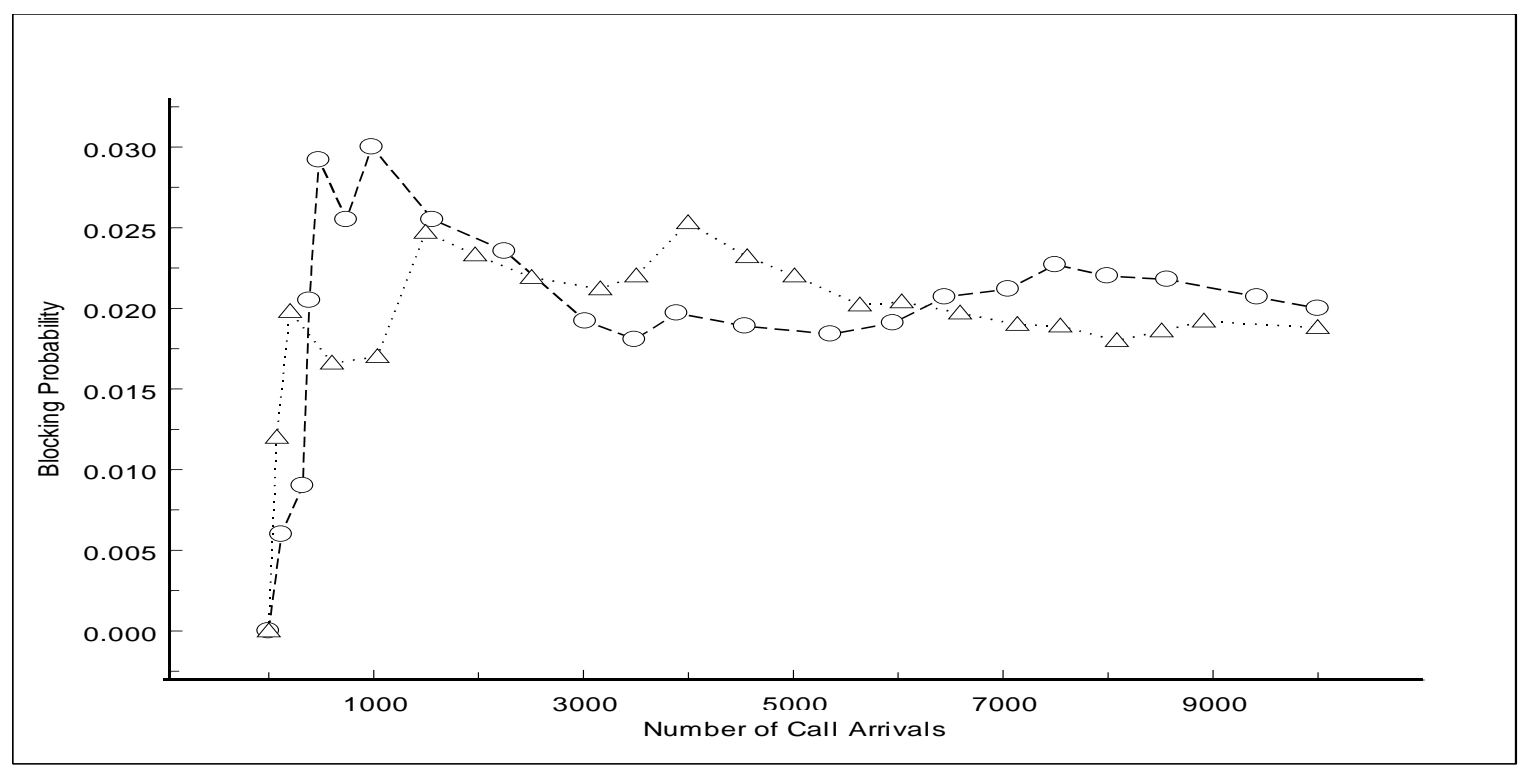

Figure 4: Blocking probability versus call arrivals for the first 10,000 call arrivals for two different simulation runs with different random numbers.

\section{REFERENCES}

[1]K. Pawlikowski, H. D. Jeong, and J. S. Lee, "On credibility of simulation studies of telecommunication networks," IEEE Communications Magazine, vol. 40, pp. 132-9, 2002.

[2]R. W. Conway, "Some tactical problems in digital simulation," Management Science, pp. 47-61, 1963.

[3]K. Pawlikowski, "Steady state simulation of queueing processes: survey of problems and solutions," ACM Computing Surveys, pp. 123-170, 1990.

[4] R. Madansky, "Optimal Initial Condition for a Simulation Problem," Operations Research, pp. 570-577, 1976.

[5]G. S. Fishman, "Grouping Observations in Digital Simulation," Management Science, pp. 510-521, 1978.

[6]P. T. Strait, Probability and Statistics with Applications. New York: Harcourt Brace Jovanovich inc, 1983.

[7] G. Bancroft and G. O'sullivan, Maths and Statistics. Berkshire, UK: McGraw Hill Book Company, 1998.

[8]R. Barrett, Mathematics with Statistics. Auckland: ESA Publications New Zealand, 1996.

[9]G. N. Higginbottom, Performance Evaluation of Communication Networks. Boston and London: Artech House 1998.

[10]S. S. Kolahi, "Impact of propagation parameters on neighbouring cells interference in power controlled CDMA systems," IEEE Tencon '05, Melbourne, 2005.

[11]S. S. Kolahi, A. G. Williamson, and K. W. Sowerby, "Other-cell interference in CDMA systems," Electronics Letters, vol. 40, pp. 1134-1135, 2004.

[12]J. S. Evans and D. Everitt, "On the teletraffic capacity of CDMA cellular networks," IEEE Transactions on Vehicular Technology, vol. 48, pp. 153-165, 1999.

[13]D. D. Lee, D. H. Kim, Y. J. Chung, H. G. Kim, and K. C. Whang, "Other cell interference with power control in macro/microcell CDMA networks," IEEE Vehicular Technology Conference, vol. 2, pp. 1120-1124, 1996.

[14]A. M. Viterbi and A. J. Viterbi, "Erlang capacity of a power controlled CDMA system," IEEE Journal on Selected Areas in Communications, vol. 11, pp. 892-900, 1993.
[15]K. S. Gilhousen, I. M. Jacobs, R. Padovani, A. J. Viterbi, L. A. Weaver, and C. E. Wheatley, "On the capacity of a cellular CDMA system," IEEE Transactions on Vehicular Technology, vol. 40, pp. 303-312, 1991.

[16]S. S. Kolahi, "Other cells interferences of power controlled CDMA systems," 2002 IEEE International Conference on Personal Wireless Communications (ICPWC2002), pp. 319-321, 2002.

[17] L. Kleinrock and R. Gaid, Queueing Systems, Problems and Solutions. Los Angles, USA: John Wiley and Sons Inc, 1996.

[18] L. Kleinrock, Queueing Systems, Volume II: Computer Applications. New York and London: John Wiley \& Sons, 1975.

[19] J. F. Hayes and T. Babu, Modeling and Analysis of Telecommunications Networks. Hoboken, NJ: John Wiley \& Sons, 2004.

[20] L. Kleinrock, Queueing Systems, Volume I: Theory. New York and London: John Wiley \& Sons, 1975.

[21] S. S. Kolahi, "On Erlang capacity of CDMA systems," in Mobile and Wireless Communications, C. G. Omidyar, Ed. Norwell, MA: Kluwer Academic Publishers, pp. 227-232, 2003.

[22] S. S. Kolahi, A. G. Williamson, and K. W. Sowerby, "Sensitivity analysis of the reverse link Erlang capacity of CDMA systems," 2004 IEEE Wireless Communications and Networking Conference, WCNC'04, pp. 2220-2225, 2004.

[23] S. S. Kolahi, Traffic Dimensioning of Cellular Systems Using Simulation- A simulation Guide for Cellular Systems, Lambert Academic Publishers, Germany, 2010.

[24] S.S. Kolahi, Analysis of Results in Simulation and Modelling of CDMA Systems, $12^{\text {th }}$ IEEE Symposium on Computers and Communications, ISCC 2007, pp. 679-684. 